\title{
Why South Africa urgently needs to support the development of pregnancy exposure registries
}

Recent safety concerns regarding the newly introduced first-line HIV integrase inhibitor dolutegravir (DTG) have brought necessary attention to the need for pharmacovigilance during pregnancy and breastfeeding. In 2018, Botswana's Tsepamo study ${ }^{[1]}$ identified a signal of increased risk of neural tube defects (NTDs) in infants exposed to DTG periconception. This finding prompted a flurry of response in those countries where DTG is or will be a cornerstone of first-line antiretroviral regimens. ${ }^{[2]}$ Previously, 12 years passed from the first case reports of suspected efavirenz teratogenicity to the acquisition of sufficient observational data to allay these fears. ${ }^{[3,4]}$

Pregnant women are increasingly exposed to medicines and vaccines. In the USA, more than $90 \%$ of women report taking at least one prescription or over-the-counter medicine during pregnancy. ${ }^{[5]}$ In most cases the safety of these products in pregnancy is unknown. In Africa, women of child-bearing potential (WOCBP) are at the heart of the HIV, malaria and tuberculosis (TB) epidemics and are exposed to new medicines with limited data on safety in pregnancy.

South Africa (SA) will soon introduce DTG as part of firstline antiretroviral therapy, with strong caution for WOCBP to use effective contraception, and will have the largest number of women using DTG in the world. Pregnancies will occur even if contraceptive services are enhanced. Determining whether the DTG risk signal is real or spurious requires ongoing collection of prospective data. To this end, prospective pregnancy exposure registries (PERs) are being developed at sentinel sites in the public sector. A PER was first implemented in the eThekwini district of KwaZulu-Natal (KZN) Province in 2013 and subsequently in the Klipfontein/Mitchell's Plain subdistricts in Western Cape Province in 2016. Both sites adapted the World Health Organization (WHO)recommended approach of prospective data collection on exposures in a cohort of pregnant women from the first antenatal visit until the time of delivery. ${ }^{[6]}$

The National Institutes of Health-funded Tsepamo study in Botswana ${ }^{[1]}$ similarly adapted WHO recommendations and included the following crucial elements to maximise validity and support causal inference:

- Exposure data prospectively recorded throughout antenatal care (possibly captured later from the patient-held record)

- A control group of HIV-uninfected women, allowing for concurrent establishment of local background rates of congenital anomalies, including NTDs

- Exposures to non-default antiretroviral regimens, allowing for additional comparisons

- Surveillance embedded in routine antenatal and perinatal services, with data collected from routine clinical records

- Congenital malformations identified through standardised surface examinations on all live and stillbirths, verified (remotely) by a clinical geneticist, using photographs

- Independent of pharmaceutical industry involvement.

Ongoing monitoring and collection of information on additional exposures in the Botswana cohort have, reassuringly, failed to identify any additional cases. ${ }^{[7]}$

This approach facilitates the collection of all pharmaceutical exposures, affording the opportunity to assess the safety of other treatments commonly used in the same cohort of women (TB medicines, antiepileptics, vaccinations, etc.). It avoids the creation of research silos, establishing instead a platform for investigating the safety of medicines used by WOCBP, while restricting data elements to those collected as part of routine obstetric and neonatal care. It does, however, depend on quality care and clinical record keeping, which requires strengthening of these areas, particularly the recording of antenatal exposures and newborn surface examinations (including of stillbirths).

\section{Regulatory actions}

While awaiting further data, SA has made regulatory, clinical and policy recommendations on the use of DTG in WOCBP. In approving the registration of DTG-containing fixed-drug combinations, the South African Health Products Regulatory Authority (SAHPRA) mandated all manufacturers of DTG-based fixed-dose combinations to ensure that clinicians have access to reporting forms to refer all DTG-exposed pregnancies (regardless of outcome) to the international Antiretroviral Pregnancy Register (APR), an initiative that collates global pregnancy exposure case reports and compares and reports regularly on birth defect rates across antiretroviral therapy exposures. In addition, all suspected adverse reactions, particularly those not clearly reflected in the product information, must be reported to SAHPRA through its adverse reaction reporting system. ${ }^{[8]}$ These are essential components of a more comprehensive risk management plan being implemented for DTG. More generally, SAHPRA will need to develop guidelines and tools to strengthen PERs to promote the effective monitoring of all health products.

\section{Current status of PER projects in SA}

Data generated by the existing PER projects will be an integral component of DTG safety monitoring. Combining the approaches of SAHPRA, the WHO and the APR registry will optimise the robustness of causality assessments.

Implementation of the PER, particularly in KZN, has been challenging. Uncertainties, lapses in funding, contention for political support in the face of competing priorities, and lack of record linkage capacity have hampered the generation of timely outputs. Many have called for less demanding alternative approaches, such as case detection of targeted congenital malformations or the establishment of a still poorly defined 'national pregnancy register'. While all options need to be considered, whatever approach is selected must ensure that questions of drug safety in pregnancy can be answered with confidence and evidence accepted as valid by national and international policymakers.

To inform guidelines for the use of DTG in WOCBP, the National Department of Health has held consultations with clinicians, researchers, SAHPRA, the WHO, and civil society organisations representing the interests of women living with HIV. There was consensus that, while SA has centres of excellence, more needs to be done to build a network of sentinel PER sites across the country using the WHO approach. Above all, the PER projects should be nested within, and supported and informed by, the national maternal and child health programmes.

Urgent delivery of a more effective PER network in SA requires commitment, collaboration and investment from all stakeholders. Such support would include sustained political support, the 
investment of national resources, and rigorous ethical and scientific review to ensure that the safety of priority health products is well understood, regardless of the impact of that information on treatment policies, economics and logistics. The benefits of such an initiative will extend far beyond addressing the DTG concerns, in pursuit of quality care for pregnant women and their infants.

\section{Ushma Mehta, Emma Kalk}

Centre for Infectious Disease Epidemiology and Research, School of Public Health and Family Medicine, Faculty of Health Sciences, University of Cape Town, South Africa

ushma.mehta@uct.ac.za

\section{Lee Fairlie}

Wits Reproductive Health and HIV Institute, University of the Witwatersrand, Johannesburg, South Africa

\section{Andrew Boulle}

Centre for Infectious Disease Epidemiology and Research, School of Public Health and Family Medicine, Faculty of Health Sciences, University of Cape Town, South Africa; and Health Impact Assessment, Department of Health, Provincial Government of the Western Cape, Cape Town, South Africa

\section{Helen Rees}

Wits Reproductive Health and HIV Institute, University of the Witwatersrand, Johannesburg, South Africa

\footnotetext{
1. Zash R, Makhema J, Shapiro RL. Neural-tube defects with dolutegravir treatment from the time of conception. N Engl J Med 2018;379(10):979-981. https://doi.org/10.1056/NEJMc1807653

2. Nakkazi E. Changes to dolutegravir policy in several African countries. Lancet 2018:391:2296. 2. Nakkazi E. Changes to dolutegravir policy in
https://doi.org/10.1016/S0140-6736(18)31641-6

3. Ford N, Mofenson L, Shubber Z, Calmy A, et al. Safety of efavirenz in the first trimester of pregnancy: An updated systematic review and meta-analysis. AIDS 2014;28(Suppl 2):S123-S131. https://doi.org/10.1097/QAD.0000000000000231

4. Black V, Schwartz SR. Issues about periconception use of dolutegravir are reminiscent of early concerns about efavirenz. Lancet HIV 2018;5(12):e732-e736. https://doi.org/10.1016/S23523018(18)30249-2

5. Mitchell AA, Gilboa SM, Werler MM, Kelley KE, Louik C, Hernandez-Diaz S. Medication use during pregnancy, with particular focus on prescription drugs: 1976 - 2008. Am J Obstet Gynecol 2011;205(1):51.e1-51.e8. https://doi.org/10.1016/j.ajog.2011.02.029

6. Mehta U, Clerk C, Allen E, et al. Protocol for a drugs exposure pregnancy registry for implementation in resource-limited settings. BMC Pregnancy Childbirth 2012;12:89. https://doi.org/10.1186/1471in resource-1

7. Zash R, Holmes L, Makhema J, et al. Surveillance for neural tube defects following antiretroviral exposure from conception. Presented at symposium on 'Safety of dolutegravir in pregnancy', 22nd International AIDS Conference (AIDS 2018), Amsterdam, 23 - 27 July 2018. http://www.natap. org/2018/IAC/IAC_52.htm (accessed 1 April 2019).

8. National Department of Health, South Africa/Medicines Control Council. Adverse drug reaction reporting form. https://www.sahpra.org.za/documents/86422f1b6.04_ARF1_Jul16_v4.pdf (accessed 20 February 2019).
}

S Afr Med J 2019;109(5):294-295. DOI:10.7196/SAMJ.2019.v109i5.14008 